\title{
Toxicity of newer versus older antidepressants
}

\author{
John A. Henry
}

Of all the controversies over the different medications used in psychiatry, there is probably least dispute about the effectiveness of antidepressants in controlling depressive illness. However, the choice of which antidepressant to use is bedevilled by the spectre of potential toxicity from adverse effects, interactions and overdose. The unwanted effects may vary from mild and tolerable to potentially lethal. At one time it was relatively easy to become familiar with the problems surrounding the original two main groups of antidepressants the tricyclic antidepressants (TCAs) and the monoamine oxidase inhibitors (MAOIs). Recent years have seen the introduction of a number of newer drugs, with a wide range of chemical structures and differing pharmacological activities. They also have a new spectrum of adverse effects and interactions. It is worth reviewing the major differences between old and new antidepressants, and to identify the areas in which clinical caution must be exercised in order to avoid pitfalls and maximise clinical benefit.

\section{Adverse effects}

No drug is free from adverse effects. Those of the TCAs are well known, and can constitute relative contraindications for a number of different individuals and illnesses. The most important limitations are prostatic obstruction, narrow-angle glaucoma and some cardiac conditions. Most of the TCAs are contraindicated within three months of a myocardial infarction or in patients under treatment for heart failure. These drugs are also more likely to cause postural hypotension in the elderly. However, the newer drugs also produce adverse effects, which may limit their use in certain circumstances (Box 1). Lofepramine can be considered a newer tricyclic drug and has milder anticholinergic effects than the other TCAs. The most common adverse effects caused by the different antidepressants are compared in Table 1. Other toxic effects are uncommon. Rashes occur with most drugs, including fluoxetine, and liver damage can occur with the TCAs and lofepramine.

\section{Adverse effects and compliance}

A major issue concerning adverse effects of antidepressants is their effect on compliance, which may limit the effectiveness of the treatment and thus prolong the depression, leaving the individual at risk of suicide. In addition the unused tablets are available for overdose. One evaluation of patients seen in general practice, attending psychiatric out-patient clinics and participating in antidepressant drug trials, revealed that between 32 and $64 \%$ of noncompliant patients defaulted because of sideeffects, and some took no medication because they anticipated side-effects (Johnson, 1986). The potential of different antidepressants to cause side-effects varies greatly. However, tolerance occurs to many of the adverse effects of antidepressants. The anticholinergic effects of TCAs can be diminished by starting at a lower than therapeutic dose. Tolerance soon occurs to the nausea caused by the selective serotonin reuptake inhibitors (SSRIs). The tolerability of SSRIs and TCAs in terms of adverse events has been compared in meta-analyses and these have been reviewed elsewhere in this issue (Anderson, 1997).

Dr John Henry is a Consultant Physician at Guy's Hospital and at the Medical Toxicology Unit, Guy's Hospital, London SE1 9RT. He has a special interest in the mechanisms and epidemiology of antidepressant toxicity, and in the complications caused by drugs of abuse. 
Box 1. Adverse effects of antidepressants

Tricyclics

Dry mouth, blurred vision, constipation, urinary retention, drowsiness, postural hypotension

Moclobemide

Insomnia, dizziness, nausea, headache, restlessness, agitation, confusion

Monoamine oxidase inhibitors

Dry mouth, blurred vision, constipation, urinary retention, drowsiness (sometimes insomnia), tremor, dizziness, weakness, fatigue, gastrointestinal problems

Selective serotonin reuptake inhibitors

Citalopram: nausea, sweating, tremor, drowsiness, dry mouth

Fluoxetine: nausea, headache, nervousness, insomnia, anxiety, dizziness, weakness

Fluvoxamine: nausea, vomiting, drowsiness, diarrhoea, agitation, tremor, hypokinesia, asthenia

Paroxetine: constipation, insomnia, dry mouth, tremor, weakness, sweating, nausea, drowsiness, headache

Sertraline: dry mouth, nausea, diarrhoea, tremor, sweating, dyspepsia, ejaculatory delay

Nefazodone

Weakness, dry mouth, nausea, dizziness, drowsiness

Venlafaxine

Nausea, headache, drowsiness, insomnia, dry mouth, dizziness, constipation, weakness, nervousness

\section{Interactions}

While drug interactions are liable occur with all antidepressants, some groups of drug are more prone to interactions than others. The 'cheese' interaction with the older MAOIs is very well known, and consists of a hypertensive crisis provoked by certain foods and drugs. This interaction is much less likely to occur with the newer selective inhibitors of monoamine oxidase A, such as moclobemide, and restriction of diet is not required provided the patient does not indulge in binges of contraindicated foods. Some of the SSRIs may increase the adverse effects of lithium, especially fluoxetine, and although the TCAs are normally well tolerated with lithium there may rarely be myoclonus and seizures.

Since many of the newer drugs are serotoninergic, the possibility of provoking a serotonin syndrome by simultaneously prescribing drugs with a serotoninergic mode of action (the five SSRIs, clomipramine, nefazodone and venlafaxine) needs to be considered carefully. In most cases this involves introducing the drug gradually by titrating the dose upwards and telling the patient not to take any further doses if they experience any untoward symptoms. The syndrome presents as progressive restlessness, hyper-reflexia, shivering, tremor and sweating due to an excess of serotonin at a synaptic level.Agitation, confusion and hypomanic behaviour may occur. The pupils are usually widely dilated. If muscle spasms are severe or the patient develops a pyrexia, urgent medical attention will be required. Drugs such as propranolol or cyproheptadine may be used for their antiserotoninergic effect (Brown et al, 1996). In extreme cases paralysis with muscle relaxants and mechanical ventilation may be required. Since the syndrome includes muscle stiffness and rigidity, restlessness, agitation and a raised temperature, it needs to be considered in the differential diagnosis of neuroleptic malignant syndrome (Sternbach, 1991).

Another type of interaction which must be taken into account is the oxidative enzyme inhibiting properties of the SSRIs. Several of these drugs have the capacity to inhibit hepatic metabolism of drugs which the patient may be receiving. The plasma level of some TCAs may be raised by this mechanism. SSRIs such as fluvoxamine and sertraline can prolong the prothrombin time by inhibiting the metabolism of warfarin. Paroxetine also prolongs prothrombin time, but in addition may further increase the tendency to bleeding; the reason for this is not fully established. This drug should, therefore, be used with extreme caution in any patient on warfarin.

\section{Changing between antidepressants}

Quite frequently the antidepressant first chosen does not produce the desired effect, and treatment 


\section{Table 1. More common adverse effects of different antidepressant drugs compared}

\begin{tabular}{|llllll|}
\hline Drug & $\begin{array}{l}\text { Anticholin- Sedation } \\
\text { ergic effect }\end{array}$ & $\begin{array}{l}\text { Stimulant Nausea } \\
\text { effect }\end{array}$ & $\begin{array}{l}\text { Convulsant } \\
\text { effect }\end{array}$ & $\begin{array}{l}\text { Cardiac } \\
\text { effects }^{1}\end{array}$ & $\begin{array}{l}\text { Toxicity in } \\
\text { overdose }\end{array}$ \\
\hline
\end{tabular}

\begin{tabular}{|c|c|c|c|c|c|c|c|}
\hline \multicolumn{8}{|c|}{ Tricyclic antidepressants } \\
\hline Amitriptyline & +++ & +++ & 0 & + & ++ & +++ & +++ \\
\hline Amoxapine & +++ & + & 0 & 0 & +++ & + & +++ \\
\hline Clomipramine & ++ & + & 0 & + & ++ & ++ & ++ \\
\hline Desipramine & + & + & 0 & + & + & ++ & ++ \\
\hline Dothiepin & ++ & ++ & 0 & 0 & ++ & ++ & +++ \\
\hline Doxepin & ++ & ++ & 0 & 0 & ++ & ++ & ++ \\
\hline Imipramine & +++ & ++ & 0 & + & ++ & ++ & +++ \\
\hline Lofepramine & + & + & 0 & 0 & 0 & + & 0 \\
\hline Nortriptyline & ++ & ++ & 0 & + & + & ++ & ++ \\
\hline Protriptyline & ++ & + & 0 & + & + & + & ++ \\
\hline Trimipramine & +++ & ++ & 0 & 0 & + & ++ & ++ \\
\hline \multicolumn{8}{|c|}{ Monoamine oxidase inhibitors (MAO) } \\
\hline Iproniazid & 0 & 0 & ++ & + & 0 & 0 & ++ \\
\hline Isocarboxazid & 0 & 0 & + & + & 0 & + & ++ \\
\hline Phenelzine & 0 & 0 & + & + & 0 & 0 & ++ \\
\hline Tranylcypromine & 0 & 0 & +++ & + & 0 & 0 & ++ \\
\hline \multicolumn{8}{|c|}{ Reversible inhibitors of monoamine oxidase A (RIMA) } \\
\hline Moclobemide & 0 & 0 & + & + & 0 & 0 & + \\
\hline \multicolumn{8}{|c|}{ Selective serotonin reuptake inhibitors (SSRIs) } \\
\hline Citalopram & 0 & 0 & 0 & ++ & 0 & + & + \\
\hline Fluvoxamine & 0 & + & 0 & +++ & + & 0 & 0 \\
\hline Fluoxetine & 0 & + & ++ & 0 & 0 & 0 & \\
\hline Sertraline & 0 & 0 & 0 & ++ & 0 & 0 & 0 \\
\hline \multicolumn{8}{|l|}{ Atypical drugs } \\
\hline Maprotiline & ++ & ++ & 0 & + & +++ & ++ & +++ \\
\hline Mianserin & 0 & +++ & 0 & 0 & 0 & 0 & 0 \\
\hline Nefazodone & 0 & 0 & 0 & ++ & 0 & 0 & $\mathrm{i}$ \\
\hline Trazodone & 0 & ++ & 0 & +++ & 0 & + & + \\
\hline Venlafaxine & 0 & 0 & 0 & ++ & $\mathrm{i}$ & 0 & $\mathrm{i}$ \\
\hline Viloxazine & + & + & 0 & ++ & 0 & 0 & 0 \\
\hline
\end{tabular}

1. Safety of administration in patients with heart problems (angina, arrhythmias, etc.).

Key: +++ , very strong; ++ , strong; + , mild; 0 , mild or absent; $i$, insufficient information.

needs to be changed to another drug. When introducing a new drug, the question of antidepressant-antidepressant interactions must be considered. This problem is compounded by the long elimination half-lives of some antidepressants, particularly fluoxetine, and the time taken for the pharmacological effect to disappear, which is particularly notable in the case of the older MAOIs. One exception is moclobemide, which in most cases requires no treatment-free interval when introducing a new drug, because its persistence in the body is short. Thus, the time factor deserves close consideration, particularly when changing antidepressants, especially with drugs such as fluoxetine, which has a long persistence in the body (up to 35 days; a seven-day interval is usually sufficient for other SSRIs, trazodone, nefazodone and venlafaxine) and the older MAOIs (interactions may occur up to 14 days after discontinuation).

\section{Adverse effects and undertreatment}

An important problem, especially in the field of general practice, is the danger of underprescribing in order to reduce adverse effects. While there is no doubt that most adverse effects are dosedependent and therefore will be reduced by prescribing a lower dose of drug, it is also apparent that therapeutic levels of the drug may not be reached, thus resulting in treatment failure. 
Subtherapeutic dosing, particularly with the older TCAs, is common in general practice in an attempt to avoid the side-effects attendant on higher doses, and is liable to prejudice treatment outcome (Thompson \&Thompson, 1989). A recent survey of 500 general practices in the UK based on over 70000 antidepressant prescriptions has shown that $61 \%$ were for amitriptyline, dothiepin and clomipramine, and that of these only $13.4 \%$ were prescribed at effective therapeutic doses (defined as $125 \mathrm{mg}$ /day or more; Donoghue, 1994).

\section{Fatal toxicity from antidepressant overdose}

A number of studies have shown that drugs and drug groups can be ranked in terms of the number of deaths per million prescriptions (see Henry et al, 1995). When such a 'fatal toxicity index' is constructed, the older TCAs are the most lethal in overdose, the MAOIs have intermediate toxicity, and the newer drugs, which include the SSRIs, have lowest toxicity in overdose. Two of the older tricyclic drugs, amitriptyline and dothiepin, were associated with $81.6 \%$ of all antidepressant overdose deaths in Britain. These figures suggest that the older TCAs are more likely to prove fatal in overdose than most of the newer drugs. Although there are several sources of bias in these data, the figures correlate with clinical impressions of overdose toxicity and with animal studies of lethality of antidepressants (Molcho \& Stanley, 1992; Kelvin \& Hakansson, 1989). There is sufficient evidence to implicate several of the older tricyclic drugs in fatal toxicity of antidepressants.

Overdose of TCAs can lead to anticholinergic effects, such as tachycardia, hallucinations and mydriasis; other pharmacological properties can cause brisk reflexes, coma and convulsions, but the most serious effects are due to the quinidine-like effects of the tricyclic drugs, which can lead to hypotension and cardiac arrhythmias and may be responsible for a fatal outcome. Both the older and newer MAOIs in overdose can produce a severe serotoninergic syndrome which may progress over 12-48 hours, leading to fatal hyperthermia unless treated. Other antidepressants in overdose generally produce milder symptoms, and the outcome is rarely fatal; however, deaths from probable cardiotoxicity of citalopram have been reported (Öström et al, 1996).

Choice of medication clearly involves considering the consequences of prescribing. Every doctor prescribing the older TCAs is confronted with the possibility that the treatment itself may prove the ultimate instrument of suicide (Meredith, 1995; Smith, 1995). Safety in overdose should be a matter for concern. A change in prescribing habits could have an important public health impact and would help contribute to the 'Health of the Nation' strategy (Department of Health, 1994; Freemantle et al, 1994).

\section{Conclusions}

The 'Defeat Depression' campaign launched in 1992 by the Royal College of Psychiatrists and the Royal College of General Practitioners aimed to improve the diagnosis and treatment of depression and is likely to result in increased prescriptions for antidepressant drugs over the next few years. While antidepressants are demonstrably effective in controlling depressive illness, they must be prescribed with care, for three main reasons: they can produce adverse effects; they may interact with other medication; and they may cause morbidity or mortality in overdose. The most notable problem about adverse effects is that they may hinder compliance or lead to subtherapeutic prescribing and thus defeat the object of treatment. Some interactions are potentially serious, and prescribers need to be aware of potential pitfalls. The older drugs have the obvious advantage of being well tried, while it takes time for experience and familiarity to develop with the newer drugs. The older drugs suffer from the limitation of adverse effects such as sedation and anticholinergic effects. Several of the newer drugs can cause initial nausea and their list of potential interactions is longer and more complex. Most of the newer antidepressants are relatively safe in overdose, but the older TCAs are demonstrably more toxic and are significantly more likely to prove fatal in overdose than other antidepressants. The prescribing psychiatrist needs to keep these considerations in mind in order to optimise treatment for a disease which is eminently treatable yet potentially lethal. Today's psychiatrist needs to be more of a physician and pharmacologist than his predecessor.

\section{References}

\footnotetext{
Anderson, I. (1997) Lessons to be learnt from meta-analyses of newer versus older antidepressants. Advances in Psychiatric Treatment, 3, 57-62.

Brown, T. M., Skop, B. P. \& Mareth, T. R. (1996) Pathophysiology and management of the serotonin syndrome. Annals of Pharmacotherapy, 30, 529-533.
} 
Donoghue, J. M. (1994) The prescribing of antidepressants in general practice: The use of PACT data. Postgraduate Medical Journal, 70 (suppl. 2), S23-S24.

Freemantle, N., House, A., Song, F., et al (1994) Prescribing selective serotonin reuptake inhibitors as strategy for prevention of suicide. British Medical Journal, 309, 250-253.

Department of Health (1994) Health of the Nation: Suicide Prevention - The Challenge Confronted. London: Her Majesty's Stationery Office.

Henry, J. A., Alexander, C. A. \& Sener, E. K. (1995). Relative mortality from overdose of antidepressants. British Medical Journal, 310, 221-224.

Johnson, D. A. W. (1986) Non-compliance with antidepressant therapy: An underestimated problem. Internal Medicine, suppl. 11, 14-17.

Kelvin, A. S. \& Hakansson, S. (1989) Comparative acute toxicity of paroxetine and other antidepressants. Acta Psychiatrica Scandinavica, 80, 31-33.

Meredith, T. (1995) Epidemiology of poisoning. Medicine, 23, 1-3.

Molcho, A. \& Stanley, M. (1992) Antidepressants and suicide risk: Issues of chemical and behavioural toxicity. Journal of Clinical Pharmacology, 12 (suppl. 2), S13-S18.

Öström, M., Eriksson, A., Thorson, J., et al (1996) Fatal overdose with citalopram. Lancet, 348, 339-340.

Smith, T. (1995) Differences between general practices in hospital admission rates for self-inflicted injury and selfpoisoning: Influence of socioeconomic factors. British Journal of General Practice, 45, 458-462.

Sternbach, H. (1991) The serotonin syndrome. American Journal of Psychiatry, 148, 705-713.

Thompson, C. \& Thompson, C. M. (1989) The prescribing of antidepressants in general practice. II: A placebo-controlled trial of low-dose dothiepin. Human Psychopharmacology, 4, 191-204.

\section{Multiple choice questions}

1 The tricyclic drugs:

a are all highly toxic in overdose

b an SSRI given simultaneously may increase plasma drug concentration

c anticholinergic effects include tachycardia and mydriasis

d are contraindicated within three months of a surgical operation

e postural hypotension may lead to falls.
2 Serotonin syndrome:

a consists of a hypertensive crisis

$b$ occurs from overdose of SSRIs

c occurs from overdose of MAOIs

d may occur if two SSRIs are given simultaneously

e the patient may have stiff muscles and a fever.

3 The following drugs should be considered highly toxic in overdose:
a amitriptyline
b moclobemide
c paroxetine
d lofepramine
e imipramine.

4 Adverse effects:

a frequently lead to subtherapeutic dosing with TCAs

b tyramine-containing foods should be avoided by patients taking trazodone

c of lofepramine are worse than those of imipramine

d venlafaxine does not cause nausea or drowsiness

e care should be taken when giving an SSRI to patients taking digoxin.

\begin{tabular}{|ccclll||}
\hline \multicolumn{2}{|c|}{ MCQ answers } & & & \\
$\mathbf{1}$ & 2 & 3 & & 4 \\
a F & a F & a & T & a T \\
b T & b F & b F & b F \\
c T & c T & c F & c F \\
d F & d T & d F & d F \\
e T & e T & e T & e F \\
\hline
\end{tabular}

